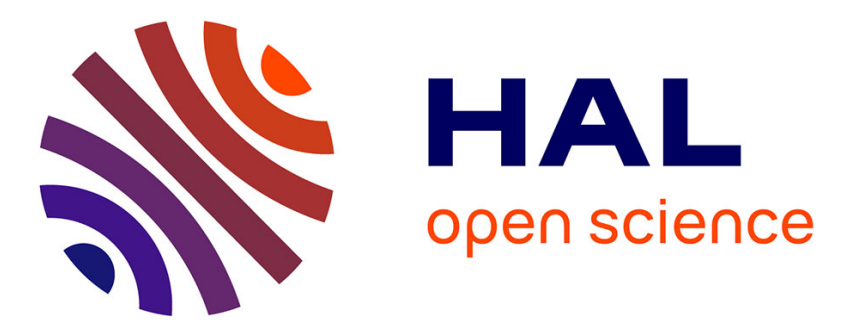

\title{
Wavelength conversion and temporal compression of a pulse train using a dispersion oscillating fibre
} \author{
Fang Feng, Julien Fatome, Yanne Kouomou Chembo, Stefan Wabnitz,
} Christophe Finot

\section{- To cite this version:}

Fang Feng, Julien Fatome, Yanne Kouomou Chembo, Stefan Wabnitz, Christophe Finot. Wavelength conversion and temporal compression of a pulse train using a dispersion oscillating fibre. Electronics Letters, 2014, 50 (10), pp.768-770. 10.1049/el.2014.0627 . hal-00980893

\section{HAL Id: hal-00980893 https://hal.science/hal-00980893}

Submitted on 19 Apr 2014

HAL is a multi-disciplinary open access archive for the deposit and dissemination of scientific research documents, whether they are published or not. The documents may come from teaching and research institutions in France or abroad, or from public or private research centers.
L'archive ouverte pluridisciplinaire HAL, est destinée au dépôt et à la diffusion de documents scientifiques de niveau recherche, publiés ou non, émanant des établissements d'enseignement et de recherche français ou étrangers, des laboratoires publics ou privés. 


\section{Wavelength conversion and temporal compression of a pulse train using a dispersion oscillating fibre}

\section{F. Feng, J. Fatome, A. Sysoliatin, Y. Chembo, S. Wabnitz} and C. Finot

We demonstrate the generation of a picosecond pulse train taking advantage of the cross gain occurring in a dispersion oscillating fibre. The resulting frequency-converted signal is detuned by more than $20 \mathrm{~nm}$ from the pump and can be temporally compressed by a factor 2 compared to the input sinusoidal pump wave.

Introduction: In order to alleviate the bandwidth limitations of optoelectronic modulations or the complexity of mode-locked fibre lasers, the generation of high repetition pulse sources has strongly benefited in the past from various strategies relying on the use of the Kerr effect of silica to convert a sinusoidal signal into a train of wellseparated optical pulses. For example, multiple-four wave mixing in anomalous dispersion fibres or nonlinear reshaping in normally dispersive fibres followed by a linear compensating segment are two efficient ways to achieve such a transformation [1]. A more recently demonstrated technique relies on the nonlinear reshaping of a sinusoidal beating into a train of Akhmediev breathers [2]. A very different approach takes advantage of the parametric gain experienced by a continuous seed under a sinusoidal pumping: the exponential and instantaneous gain leads to a very high level of amplification in the most intense areas of the sinusoidal profile of the pump whereas the signal remains quasi unamplified around its temporal minima. The efficiency of this approach has been demonstrated in a parametric amplifier [3] based on a fibre with constant longitudinal anomalous dispersion.

In this contribution, we show that other parametric processes can also be suitable for frequency conversion and pulse train generation applications. More specifically, we demonstrate that it is possible to take advantage of newly developed fibres having a longitudinally oscillating dispersion profile. Given the periodic variation of their properties, such fibres exhibits new gain regions that we here exploit [4, $5]$.

Experimental set-up: The experimental setup that has been developed is illustrated in Fig. 1. The pump is made of a continuous wave at 1540 $\mathrm{nm}$ delivered by a distributed feedback laser (DFB) that is intensity modulated by a Niobate Lithium modulator driven by a sinusoidal clock operating at a frequency of $10 \mathrm{GHz}$. The sinusoidal pump waveform is first amplified and then sliced by a second optical modulator to decrease the repetition rate of the source down to $2.5 \mathrm{GHz}$. An optical bandpass filter (OBPF) is then used to prevent excessive build-up of amplified spontaneous emission (ASE) before the light being launched in a high power erbium-doped fibre amplifier with an output power of $28 \mathrm{dBm}$ (corresponding to a peak power of $6 \mathrm{~W}$ ). The pump is combined by a $90 / 10$ coupler with a seed signal at $1563.2 \mathrm{~nm}$ delivered by a second external cavity laser that is amplified in order to reach the $\mathrm{mW}$ level. Next, pump and signal are launched in a 400-m long dispersion oscillating fibre (DOF) with the following parameters: spatial period of the sinusoidal dispersion profile $\Lambda=20 \mathrm{~m}$, peak-to-peak amplitude of the dispersion change $D_{A m p}=1.2 \mathrm{ps} / \mathrm{km} / \mathrm{nm}$, a normal average value of dispersion $D_{A v}=-0.4 \mathrm{ps} / \mathrm{km} / \mathrm{nm}$ at the pump wavelength, and nonlinear coefficient of $10 \mathrm{~W}^{-1} / \mathrm{km}^{-1}$. More details on the index profile and drawing techniques of this fibre can be found in refs. [6, 7]. At the output of the fibre, an optical bandpass filter is used to isolate the generated idler (wavelength $1518 \mathrm{~nm}$ ). The idler temporal and spectral profiles are monitored on a $70-\mathrm{GHz}$ photodiode associated with a high speed-sampling oscilloscope (bandwidth $70 \mathrm{GHz}$ ) and on an optical spectral analyser, respectively.

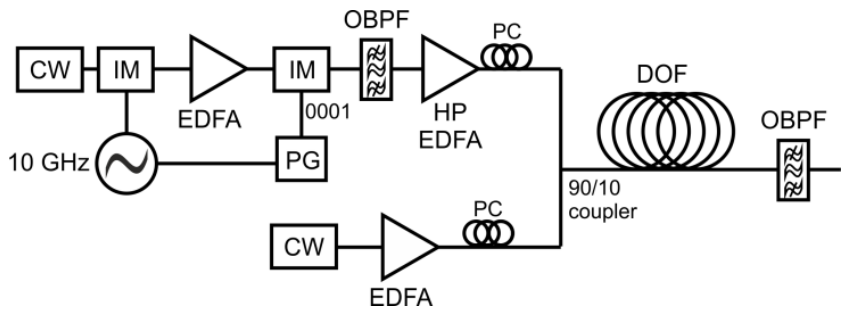

Fig. 1 Experimental setup. $C W$ : continuous wave laser ; IM : intensity modulator ; EDFA : erbium doped fibre amplifier (HP : High Power); $P G$ : pattern generator ; OBPF : optical bandpass filter ; $P C$ : polarization controller; $D O F$ : dispersion oscillating fibre.

Spectral analysis: We have plotted in Fig. 2 the initial spectrum of the pump wave launched into the fibre (dotted grey line). From the spectrum recorded at the output of the fibre in absence of filtering and seed signal, we can clearly point out the growth of resonant gain sidebands from the spontaneous amplified emission (grey solid line) $[8$, 9]. Those sidebands exhibit a gain in excess of $40 \mathrm{~dB}$, and are detuned by more than $20 \mathrm{~nm}$ from the pump that lies in the normal dispersion regime of the DOF [6]. However, those sidebands are narrower than the ones typically observed in anomalous fibres, which may limit the tunability of the conversion efficiency as well as the spectral extent of the pulse that can be processed. From the output spectrum, we can also notice the generation of additional regularly spaced sidebands, originating from multiple four wave mixing between the pump and the resonant sidebands [6]. A continuous signal seed within the resonant gain bandwidth at $1563.2 \mathrm{~nm}$ undergoes both amplification as well as spectral broadening through parametric-gain and cross-phase modulation, respectively.

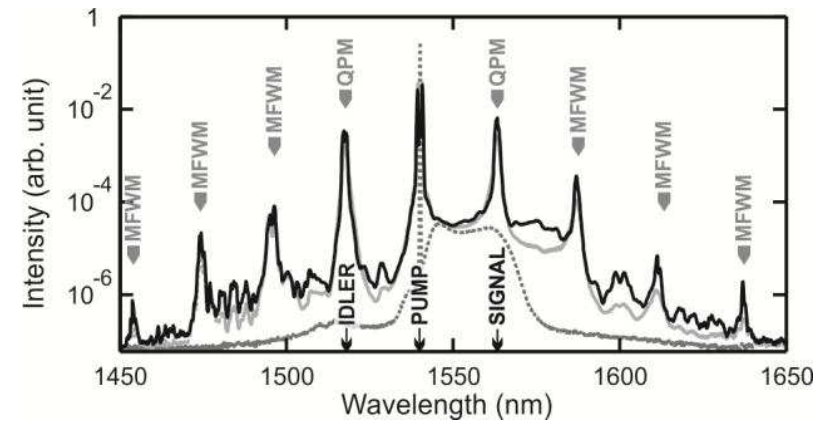

Fig. 2 Optical spectra of the pump wave (dotted dark grey line) compared with the spectra at the output of the DOF with and without seed (black and light grey solid line respectively). The gain sidebands that are linked to the resonant gain are designated by QPM whereas the ones linked to the multiple four wave mixing between the pump and the resonant sidebands are marked by MFWM.

Temporal analysis: The temporal profile of the pump pulse is plotted in Fig. $3 \mathrm{a}$ and is compared with the idler wave obtained after propagation in the DOF and optical bandpass filtering. Both saturated and unsaturated regimes are investigated and in both cases, a backgroundfree pulse train with significant temporal narrowing is observed (with temporal durations of 21 and 15 ps respectively, i.e. temporal compression factors of 1.7 and 2.4 compared to the initial duration of 37 ps). As we shall see, the regime of amplification, as well as the central wavelength of the signal, also influence the temporal waveform of the idler [10]. Indeed, the idler pulse intensity profile is close to a Gaussian shape (full grey circles) in the unsaturated amplification, whereas the idler pulse becomes parabolic-like in the saturated regime (full black diamonds). Let us also point out that the regime of operation crucially affects the stability of the converted pulse train, so that a trade-off between short duration and stability was experimentally found based on an empirical basis. Indeed, due to the exponential amplification experienced in the unsaturated regime, intensity noise of the pump converts into major fluctuations of the amplified waves [11]. On the contrary, saturation of the amplifier efficiently limits those detrimental 
shot-to-shot fluctuations as illustrated on Fig. 3b. Let us finally add that, due to the cross-phase modulation induced by the pump on the signal, the recorded pulses are not transform limited (the corresponding timebandwidth product is equal to 4 ).

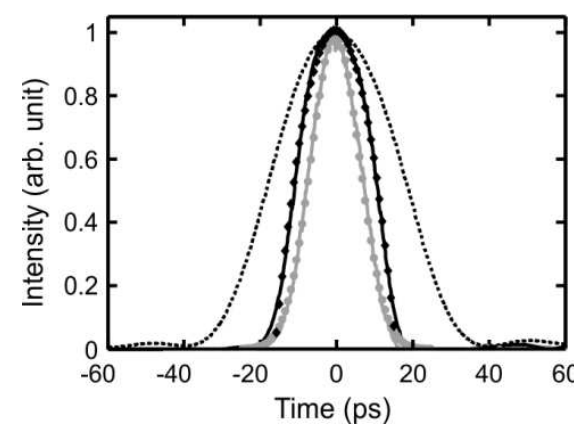

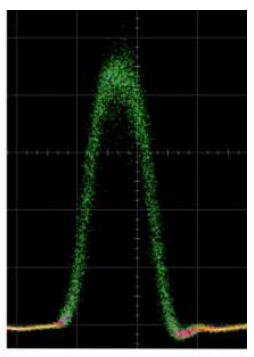

Fig. 3 a. Temporal intensity profiles of the pump (dashed black line) compared with the profile of the idler wave obtained (after OBPF) for the amplifier running in the unsaturated and saturated regimes (solid grey and black lines, respectively). Full grey circles and full black diamonds represent a Gaussian and parabolic fits of the temporal intensity profile respectively.

b. Oscilloscope trace in persistent mode of the idler wave for amplifier running in the saturated regime.

Conclusion: We have demonstrated in this contribution that the wavelength-conversion/generation of $\mathrm{GHz}$ trains of picosecond pulses through parametric gain is not restricted to the usual case of parametric amplification in a fibre with anomalous dispersion. Using a pump lying in the normal dispersion regime is also possible, thanks to a resonant quasi-phase matched process induced by the periodic sinusoidal modulation of the dispersion profile. When compared with other possible schemes such as phase-matching induced by the fourth-order dispersion coefficient, we expect that our proposed technique is more tolerant with respect to longitudinal stochastic fluctuations of the fibre properties. We also believe that improvements in the drawing process may enable in the future shorter spatial periods of the dispersion oscillation, so that larger wavelength shifts would become feasible.

Acknowledgments: We acknowledge the financial support of the Conseil Regional de Bourgogne (Pari Photcom) and the funding of the Labex ACTION program (ANR-11-LABX-01-01). The experimental work has benefited from the PICASSO Platform of the University of Burgundy. This research was funded by Fondazione Cariplo, grant n.2011-0395. A.S. acknowledges support from a Landau NetworkCariplo Foundation fellowship and the Russian Foundation for Basic Research, project no. 14-02-00856.

F. Feng, J. Fatome and C. Finot (Laboratoire Interdisciplinaire Carnot de Bourgogne, UMR 6303 Université de Bourgogne-CNRS, 9 av. A. Savary, 21078 Dijon Cedex, FRANCE)

A. Sysoliatin (Fiber Optics Research Center, 11933 Moscow, Russia)

Y.K. Chembo (FEMTO-ST/Optics department, UMR 6174 CNRSUniversity of Franche-Comté, 16 route de Gray, 25030 Besançon Cedex, France)

S. Wabnitz (Dipartimento di Ingegneria dell'Informazione, Università degli Studi di Brescia, via Branze 38, 25123, Brescia, Italy)

E-mail: christophe.finot@u-bourgogne.fr

\section{References}

1. Pitois, S., Finot, C., Fatome, J., and Millot, G., 'Generation of 20-Ghz Picosecond Pulse Trains in the Normal and Anomalous Dispersion Regimes of Optical Fibers', Opt. Commun., 2006, 260, (1), pp. 301-306.

2. Fatome, J., Kibler, B., and Finot, C., 'High-Quality Optical Pulse Train Generator Based on Solitons on Finite Background', Opt. Lett., 2013, 38, (10), pp. 1663-1665.

3. Hansryd, J. and Andrekson, P.A., 'Wavelength Tunable 40 Ghz Pulse Source Based on Fibre Optical Parametrc Amplifier', Electron. Lett., 2001, 37, (9), pp. 584-585.
4. Smith, N.J. and Doran, N.J., 'Modulational Instabilities in Fibers with Periodic Dispersion Management', Opt. Lett., 1996, 21, p. 570.

5. Droques, M., Kudlinski, A., Bouwmans, G., Martinelli, G., and Mussot, A., 'Experimental Demonstration of Modulation Instability in an Optical Fiber with a Periodic Dispersion Landscape', Opt. Lett., 2012, 37, (23), pp. 4832-4834.

6. Finot, C., Fatome, J., Sysoliatin, A., Kosolapov, A., and Wabnitz, S., 'Competing Four-Wave Mixing Processes in Dispersion Oscillating Telecom Fiber', Opt. Lett., 2013, 38, (24), pp. 5361-5364.

7. Bogatyrev, V.A., Bubnov, M.M., Dianov, E.M., Kurkov, A.S., Mamyshev, P.V., Prokhorov, A.M., Rumyantsev, S.D., Semenov, V.A., Semenov, S.L., Sysoliatin, A.A., Chernikov, S.V., Gur'yanov, A.N., Devyatykh, G.G., and Miroshnichenko, S.I., 'A Single-Mode Fiber with Chromatic Dispersion Varying Along the Length', J. Lightw. Technol., 1991, 9, (5), pp. 561-565.

8. Droques, M., Kudlinski, A., Bouwmans, G., Martinelli, G., and Mussot, A., 'Dynamics of the Modulation Instability Spectrum in Optical Fibers with Oscillating Dispersion', Phys. Rev. A, 2013, 87, (1), p. 013813.

9. Armaroli, A. and Biancalana, F., 'Tunable Modulational Instability Sidebands Via Parametric Resonance in Periodically Tapered Optical Fibers', Opt. Express, 2012, 20, (22), pp. 25096-25110.

10. Vedadi, A.A., Shoaie, M.A., and Brès, C.-S., 'Experimental Investigation of Pulse Generation with One-Pump Fiber Optical Parametric Amplification', Optics Express, 2012, 20, (24), pp. 27344-27354.

11. Hammani, K., Finot, C., and Picozzi, A., 'Extreme Statistics in Raman Fiber Amplifier : From Analytical Description to Experiments', Opt. Commun., 2011, 284, pp. 2594-2603. 